\title{
NCCN Guidelines Update for Multiple Myeloma
}

Presented by Kenneth C. Anderson, MD

\section{Abstract}

The updated NCCN Guidelines for Multiple Myeloma feature several changes in the diagnosis and management of this disease. Criteria for treatment initiation have been broadened, new staging has been recommended, and new classes of agents and combinations are included as recommended treatment. Monoclonal antibodies are among the expanded therapeutic choices, along with a histone deacetylase inhibitor and third-generation proteosome inhibitor, and there is excitement about exploring new immune-based approaches.

J Natl Compr Canc Netw 2016;14(5.5):675-677

Over the past decade, the treatment of multiple myeloma has been revolutionized by new drug approvals. Specifically, during the past 12 years, 16 treatments have been approved, including 7 new treatments in 2015 alone.

"These advances have expanded survival 3- to 4-fold, but there is still much to do," said Kenneth C. Anderson, MD, Kraft Family Professor of Medicine at Harvard Medical School, Dana-Farber Cancer Institute, and Chair of the NCCN Guidelines Panel for Multiple Myeloma.

The updated NCCN Guidelines for Multiple Myeloma broaden the criteria for treatment initiation to include asymptomatic patients, including those who lack calcium elevation, renal insufficiency, anemia, or bone abnormalities (CRAB).

"Previously, treatment has demanded CRAB abnormalities," Dr. Anderson noted. "Now, patients who have bone marrow plasmocytosis of $60 \%$ or greater; a free light-chain ratio abnormality greater than 100; or bone lesions detected on sensitive imaging are eligible for treatment even if they do not have CRAB features.

Presented by Kenneth C. Anderson, MD, Dana-Farber Cancer Institute, Boston, Massachusetts.

Dr. Anderson has disclosed that he receives other financial benefit from Acetylon Pharmaceuticals, Inc.; C4 Therapeutics, Inc.; and OncoPep.

$\mathrm{He}$ is a scientific advisor for Celgene Corporation; Gilead Sciences, Inc.;

Millennium Pharmaceuticals, Inc.; and Takeda Pharmaceuticals North

America, Inc.

Correspondence: Kenneth C. Anderson, MD, Dana-Farber Cancer

Institute, 450 Brookline Avenue, Boston, MA 02215.

E-mail: kenneth.anderson@dfci.harvard.edu
We used to wait for CRAB complications to develop, but now we can treat these patients sooner. That is a very important difference."

Many protocols are currently being studied in smoldering myeloma, with the goal of preventing or delaying progression to active myeloma. "The standard of care is to follow expectantly off all therapy, but patients with smoldering disease should now consider these clinical trials," Dr. Anderson told the audience.

Another major update to the NCCN Guidelines is a revised staging system. Traditionally, the International Staging System (ISS) has been used, which is based on serum albumin and beta- 2 microglobulin. The Revised ISS uses these factors in addition to serum lactic dehydrogenase and evidence of high-risk cytogenetics on fluorescence in situ hybridization. "The classification system is a moving target along with our advances in therapy, and I think it will undergo continued evolution. Genetics are included in the ISS for the first time," he stated.

The guidelines in the future will incorporate more stringent response criteria for minimal residual disease. "Either gene sequencing or immunophenotypic analysis multiparametric flow can detect 1 myeloma [cell] in 1 million cells," he noted.

\section{Updated Therapeutic Choices}

New to the updated NCCN Guidelines is the inclusion of lenalidomide/bortezomib/dexamethasone as a cat- 
egory 1 preferred regimen for newly diagnosed patients, regardless of whether they are candidates for transplantation. This recommendation is based on a phase III randomized trial showing superiority for the triplet regimen over combination lenalidomide/ dexamethasone. ${ }^{1}$

Additionally, for the first time an all-oral triplet, lenalidomide/ixazomib/dexamethasone, has received a category $2 \mathrm{~A}$ recommendation for upfront treatment. Ixazomib is an oral proteosome inhibitor that was FDA-approved in 2015. Dr. Anderson considers having an all-oral regimen a major advance in terms of convenience for caregivers and doctors. "Triplets have synergistic activity, improving progression-free survival [PFS] and overall survival. Therefore, we recommend triplet therapy for patients with newly diagnosed disease, for transplant candidates, and for nontransplant patients, if very fit," Dr. Anderson stated.

Maintenance therapy is considered standard practice in transplant and nontransplant candidates. Preferred regimens include bortezomib, lenalidomide (category 1 ), and thalidomide (category 1 ).

"Two studies have shown that transplantation followed by lenalidomide maintenance confers almost a 2-fold advantage in PFS. ${ }^{2,3}$ We recommend this in North America," he noted. Combination novel therapies raise the question of whether transplantation will still be needed, Dr. Anderson continued. Ongoing studies are examining this question.

Another advance in the treatment of multiple myeloma is that next-generation sequencing can distinguish outcome in patients: those who are minimal residual disease (MRD) sequence-negative have better PFS compared with those who are MRD sequence-positive. $^{4}$

A Working Group is currently developing a White Paper on how best to measure MRD and how to incorporate this into clinical trials to get new drugs approved and to define its clinical utility, he said.

\section{Previously Treated Myeloma}

The updated NCCN Guidelines for Multiple Myeloma list the following regimens as having category 1 evidence: bortezomib; bortezomib/liposomal doxorubicin; carfilzomib/lenalidomide/dexamethasone; elotuzumab/lenalidomide/dexamethasone; ixazomib/ lenalidomide/dexamethasone; lenalidomide/dexa- methasone; panobinostat/bortezomib/dexamethasone; and pomalidomide/dexamethasone.

New agents included in the updated guidelines for the first time include panobinostat, a histone deacetylase (HDAC) inhibitor, and 2 recently FDA-approved monoclonal antibodies, elotuzumab and daratumumab. Panobinostat is a broad HDAC inhibitor that can block the aggresomal breakdown of proteins. The combination of panobinostat and a proteosome inhibitor can block both aggresomal and proteasomal pathways, Dr. Anderson suggested.

Carfilzomib/dexamethasone (without lenalidomide) is another newly included combination for relapsed disease. This recommendation is based on the ENDEAVOR trial, which showed that combination carfilzomib/dexamethasone outperformed bortezomib/dexamethasone in PFS. This regimen carries little risk of neuropathy, he noted. ${ }^{5}$

"For the first time, we have monoclonal antibodies that work in multiple myeloma via several mechanisms. They mediate antibody-dependent cellular toxicity and complement-dependent cytotoxicity. Elotuzumab targets SLAMF7 and daratumumab targets CD38. Elotuzumab is approved for use with lenalidomide and dexamethasone based on data from a study demonstrating superior PFS to lenalidomide/ dexamethasone, whereas daratumumab is approved as a single agent. Daratumumab achieved a 30\% response rate in patients whose disease was resistant to bortezomib and lenalidomide," Dr. Anderson explained.

"There is still room for improvement; nevertheless we are excited about these approvals," he said.

Other avenues being explored to improve outcomes include adding a fourth agent to a triplet, such as panobinostat or daratumumab. "Immune therapies are being studied in a randomized trial where a vaccine is added to lenalidomide versus lenalidomide posttransplant to determine whether the combination increases MRD and the immune response." Additionally, Dr. Anderson said that "We now have multiple options to induce the patient's own immune response in the context of posttransplant with minimal disease. This will probably be with a combination, and it is likely to confer long-term benefit," he said. 


\section{Future Prospects}

Thinking about the future, Dr. Anderson said that immune approaches, including programmed cell death-1 (PD-1) and programmed cell death ligand-1 (PD-L1) checkpoint inhibitors, vaccines, and chimeric antigen receptor (CAR) $\mathrm{T}$ cells, hold promise for myeloma. Early studies are currently exploring these immune approaches.

Multiple myeloma has tremendous genetic heterogeneity, a point Dr. Anderson has emphasized in the past: "There are many mutations in multiple myeloma at presentation, and increased and distinct mutations at relapse. If you could achieve an immune response and a memory immune response in patients against their own myeloma, it is possible that the immune system could overcome these changes that multiple myeloma uses to evade therapy," he commented. "Immune approaches hold promise, but we need to be aware of complications."

"I hope I whet your appetite that there are new immunotherapies that can play an important new role in the myeloma treatment paradigm. In the future, novel targeted therapy combined with immune therapies will improve patient outcome even further," Dr. Anderson concluded.

\section{References}

1. Durie B, Hoering A, Rajkumar SV, et al. Bortezomib, lenalidomide, dexamethasone vs. lenalidomide and dexamethasone in patients with previously untreated multiple myeloma without an intent for immediate autologous stem cell transplant: results of the randomized phase III SWOG S0777 [abstract]. Blood 2015;126:Abstract 25.

2. Attal M, Lauwers-Cances V, Marit G, et al. Lenalidomide maintenance after stem-cell transplantation for multiple myeloma. N Engl J Med 2012;366:1782-1791.

3. McCarthy PL, Owzar H, Hofmeister CC, et al. Lenalidomide after stemcell transplantation for multiple myeloma. N Engl J Med 2012;366:11701781 .

4. Avet-Loiseau H, Corre J, Lauwers-Cances V, et al. Evaluation of minimal residual disease by next generation sequencing is highly predictive of progression free survival in the IFM/DFCI 2009 trial [abstract]. Blood 2015;126:Abstract 191.

5. Dimopoulos M, Moreau P, Palumbo A, et al. Carlfilzomib and dexamethasone versus bortezomib and dexamethasone for patients with relapsed or refractory multiple myeloma (ENDEAVOR): a randomized, phase 3, open-label, multicentre study. Lancet Oncol 2016;17:27-38. 